\title{
D-dimer testing reduced the need for ultrasonographic imaging in outpatients with suspected deep venous thrombosis
}

Wells PS, Anderson DR, Rodger M, et al. Evaluation of D-dimer in the diagnosis of suspected deep-vein thrombosis. N Engl J Med 2003;349:1227-35.

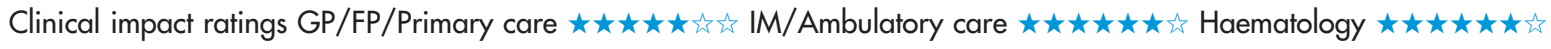

In outpatients with suspected deep venous thrombosis (DVT), does the use of D-dimer testing safely reduce the need for venous ultrasonographic imaging (VUI) and rule out DVT on the day of presentation?

\section{METHODS}

(1)

Design: randomised controlled trial

Allocation: concealed.*

Blinding: blinded (ultrasonographers, technicians who measured D-dimer concentrations, and outcome assessors).*

Follow up period: 3 months.

Setting: Thrombosis units and emergency departments of 5 academic health centres in Canada.

Patients: 1096 outpatients (mean age 58 y, 58\% women) who had suspected lower extremity DVT. Exclusion criteria included refusal or inability to consent and geographic inaccessibility.

R Intervention: patients were stratified by the application of a clinical model as "likely" or "unlikely" to have DVT and were subsequently allocated to $\mathrm{D}$-dimer testing $(n=566)$ or VUI alone $(n=530)$. Patients in the D-dimer group received VUl if they were "likely" to have DVT or if they were "unlikely" to have DVT but the D-dimer test result was positive. Patients who were "unlikely" to have DVT and whose D-dimer test result was negative did not receive VUI. Patients in the VUI alone group who were "likely" to have DVT received VUI 1 week later if the first test result was negative.

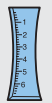

Outcomes: development of proximal DVT or pulmonary embolism in patients in whom DVT had initially been ruled out.

Patient follow up: 1082 patients (99\%) completed follow up and were included in the analysis.

*See glossary.

\section{MAIN RESULTS}

The overall prevalence of DVT or pulmonary embolism was 15.7\% during follow up. The groups did not differ for rate of proximal DVT or pulmonary embolism in patients in whom DVT had initially been ruled out (table). The mean number of ultrasonographic tests per patient was lower in the D-dimer group than in the VUI alone group $(0.78 v 1.34, \mathrm{p}=0.008)$.

\section{CONCLUSION}

In outpatients with suspected deep venous thrombosis (DVT), the use of D-dimer testing reduced the need for venous ultrasonographic

For correspondence: Professor P S Wells, Ottawa Hospital Civic Campus, Ottawa, Ontario, Canada. pwells@ohri.ca

Sources of funding: Heart and Stroke Foundation of Ontario and Heart and Stroke Foundation of Nova Scotia, Canada. imaging and ruled out DVT in patients judged clinically unlikely to have DVT without compromising safety.

Abstract and commentary also appear in ACP Journal Club.

\section{Commentary}

revious cohort studies have shown the usefulness of D-dimer testing along with an assessment of the clinical probability of disease to exclude a diagnosis of DVT. ${ }^{12}$ In this study, however, Wells et al report the first randomised trial testing a diagnostic strategy incorporating D-dimer testing, and convincingly show the value of this approach.

Whereas the study was powered to show that the rate of DVT during follow up would be $<0.8 \%$ higher in the D-dimer group than in the VUI group, the rate was actually $0.9 \%$ lower in the D-dimer group than in the VUI group, thus showing that the 2 approaches are equivalent in safety. Furthermore, fewer extremity ultrasonographic tests were done in the D-dimer group than in the VUI group. This finding implies that use of D-dimer testing may be cost saving, although this observation needs formal testing.

The authors used either of 2 assays: SimpliRED, a qualitative red cell agglutination assay, or IL-Test, an automated quantitative assay. In previous work, Kovacs et al reported negative predictive values of $96 \%$ for SimpliRED and $97 \%$ for IL-Test. ${ }^{3}$ Such high negative predictive values will not be seen in situations where the prevalence of thrombosis is higher.

This trial supports the use of D-dimer testing, after clinical assessment of the probability of thrombosis, in excluding DVT in outpatients. Results cannot be extrapolated to inpatients nor to patients suspected of having pulmonary embolism.

Jodi B Segal, MD, MPH Johns Hopkins University School of Medicine Baltimore, Maryland, USA

1 Anderson DR, Kovacs MJ, Kovacs G, et al. Combined use of clinical assessment and $\mathrm{d}$-dimer to improve the management of patients presenting to the emergency department with suspected deep vein thrombosis (the EDITED Study). J Thromb Haemos 2003;1:645-51.

2 Bates SM, Kearon C, Crowther M, et al. A diagnostic strategy involving a quantitative latex $\mathrm{D}$-dimer assay reliably excludes deep venous thrombosis. Ann Intern Med 2003:138:787-94.

3 Kovacs MJ, MacKinnon KM, Anderson D, et al. A comparison of three rapid $\mathrm{D}$-dimer methods for the diagnosis of venous thromboembolism. $\mathrm{Br} J$ Haematol 2001;115:140-4

D-dimer testing plus venous ultrasonographic imaging (VUI) $v$ VUI alone in suspected deep venous thrombosis (DVT) at 3 months*

\begin{tabular}{llll}
\hline Outcome & $\begin{array}{l}\text { D-dimer } \\
+ \text { VUI }\end{array}$ & $\begin{array}{l}\text { VUI } \\
\text { alone }\end{array}$ & $\begin{array}{l}\text { Difference } \\
\text { between } \\
\text { groups }(95 \% \mathrm{CI})\end{array}$ \\
\hline $\begin{array}{l}\text { Development of DVT or } \\
\text { pulmonary embolism in } \\
\text { patients in whom DVT had } \\
\text { been initially ruled out }\end{array}$ & $0.42 \%$ & $1.35 \%$ & $\begin{array}{l}-0.93 \% \\
(-2.2 \text { to } 0.2) \dagger\end{array}$ \\
\hline
\end{tabular}

${ }^{*} \mathrm{Cl}$ defined in glossary. †Not significant 